\title{
Multiple orthogonal polynomials, irrationality and transcendence
}

\author{
Walter Van Assche
}

Dedicated to Jerry Lange at the occasion of his 70 th birthday.

\begin{abstract}
We explain the notion of multiple orthogonal polynomials (polyorthogonal polynomials), which is closely related to Hermite-Padé rational approximation of a system of Markov functions, and give some explicit examples. As an application we show how multiple orthogonal polynomials can be used to give a constructive proof of irrationality of certain real numbers and also of transcendence of real numbers. Historically Hermite-Padé approximation was introduced by Hermite to prove the transcendence of $e$.
\end{abstract}

\section{Orthogonal polynomials}

The notion of orthogonal polynomials is an old one, going back to the previous century (Chebyshev, Stieltjes). A very good source of information is Szegö's book [Sz] and a more recent exposition can be found in [VA1]. Monic orthogonal polynomials for a positive Borel measure $\mu$ on the real line are polynomials $P_{n}$ $(n=0,1, \ldots)$ of degree $n$ and leading coefficient one such that

$$
\int P_{n}(x) x^{k} d \mu(x)=0, \quad k=0,1, \ldots, n-1 .
$$

These $n$ orthogonality conditions given $n$ linear equations for the $n$ unknown coefficients $a_{k, n}(k=0,1, \ldots, n-1)$ of the monic polynomial $P_{n}(x)=\sum_{k=0}^{n} a_{k, n} x^{k}$ (where $a_{n, n}=1$ ). This system of $n$ equations for $n$ unknowns always has a unique solution since the matrix with entries $\int x^{i+j} d \mu(x)(0 \leq i, j \leq n-1)$ (this is known as the Gram matrix) is positive definite and hence nonsingular. It is well known that such polynomials satisfy a three-term recurrence relation

$$
P_{n+1}(x)=\left(x-b_{n}\right) P_{n}(x)-a_{n}^{2} P_{n-1}(x), \quad n \geq 0,
$$

1991 Mathematics Subject Classification. Primary 42C05, 33C45; Secondary 11J72, 11J82.

Research Director of the Belgian National Fund for Scientific Research (FWO). This research is supported by FWO research project G.0278.97 and INTAS 93-219ext. 
with $P_{0}=1$ and $P_{-1}=0$. Often it is more convenient to consider the orthonormal polynomials $p_{n}(x)=\gamma_{n} P_{n}(x)$ for which

$$
\int p_{n}(x) p_{m}(x) d \mu(x)=\delta_{m, n}, \quad m, n \geq 0,
$$

so that $\gamma_{n}=\left(\int P_{n}^{2}(x) d \mu(x)\right)^{-1 / 2}>0$. The recurrence relation for these orthonormal polynomials is given by

$$
x p_{n}(x)=a_{n+1} p_{n+1}(x)+b_{n} p_{n}(x)+a_{n} p_{n-1}(x), \quad n \geq 0
$$

where

$$
a_{n}=\int x p_{n-1}(x) p_{n}(x) d \mu(x)=\frac{\gamma_{n-1}}{\gamma_{n}}, \quad b_{n}=\int x p_{n}^{2}(x) d \mu(x)
$$

A very important result in the theory of orthogonal polynomials, known as Favard's theorem, say that a system of polynomials $P_{n}(n=0,1,2, \ldots)$ satisfying a threeterm recurrence relation of the form (1.2) with $a_{n}^{2}>0$ for all $n>0$ is always a system of monic orthogonal polynomials for some positive Borel measure $\mu$ on the real line. Hence the orthogonality (1.1) and the recurrence relation (1.2) are two equivalent ways to describe monic orthogonal polynomials.

Starting from the recurrence relation (1.5) we can construct the Jacobi matrix

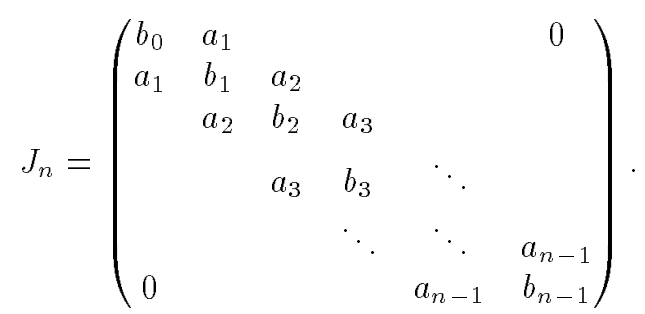

The recurrence relation (1.5) can then be written as

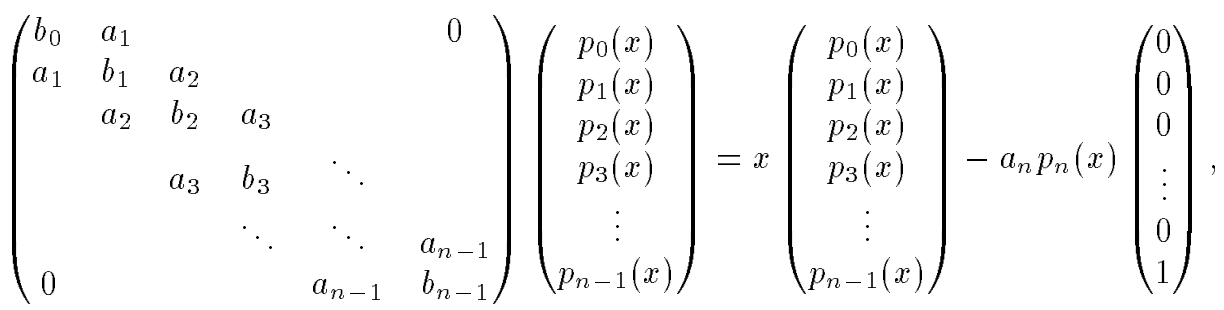

from which we conclude that every zero $x_{j, n}$ of $p_{n}$ is an eigenvalue of $J_{n}$ with eigenvector $\left(p_{0}\left(x_{j, n}\right), p_{1}\left(x_{j, n}\right), \ldots, p_{n-1}\left(x_{j, n}\right)\right)^{T}$. Since all zeros of orthogonal polynomials are simple, it follows that the eigenvalues of $J_{n}$ are the same as the zeros of $p_{n}$. One can also consider the infinite matrix $J_{\infty}$ which acts as an operator $J_{\infty}: \ell_{2} \rightarrow \ell_{2}$ on an appropriate domain. The symmetric matrix $J_{\infty}$ has selfadjoint extensions to its maximal domain (sometimes several) and the study of such a selfadjoint operator (especially the spectral theory of such operators) gives useful insight in the corresponding orthogonal polynomials.

Let us mention at this point the best known examples: the very classical orthogonal polynomials of Jacobi, Laguerre and Hermite. These three families of orthogonal polynomials satisfy a second order linear differential equation, their derivatives are again orthogonal polynomials of the same family (but with different 
parameters) and they can all be obtained from a Rodrigues formula. These properties can be extended to difference operators and $q$-difference operators and give the classical orthogonal polynomials in the Askey table [KS].

1.1. Jacobi polynomials. The monic Jacobi polynomials $\hat{P}_{n}^{(\alpha, \beta)}$ satisfy the orthogonality relations

$$
\int_{-1}^{1} \hat{P}_{n}^{(\alpha, \beta)}(x)(1-x)^{\alpha}(1+x)^{\beta} x^{k} d x=0, \quad k=0,1, \ldots, n-1,
$$

where $\alpha, \beta>-1$. There are two important differential operators, namely the lowering operator $D$ for which

$$
D \hat{P}_{n}^{(\alpha, \beta)}(x)=\left(\hat{P}_{n}^{(\alpha, \beta)}(x)\right)^{\prime}=n \hat{P}_{n-1}^{(\alpha+1, \beta+1)}(x),
$$

and the raising operator $D_{\alpha, \beta}=(1-x)^{-\alpha}(1+x)^{-\beta} D(1-x)^{\alpha}(1+x)^{\beta}$ for which

$$
\left(1-x^{2}\right) D_{\alpha, \beta} \hat{P}_{n}^{(\alpha, \beta)}(x)=-(n+\alpha+\beta) \hat{P}_{n+1}^{(\alpha-1, \beta-1)}(x) .
$$

Both (1.8) and (1.9) can be proved using integration by parts and the orthogonality (1.7). Combining the raising and lowering operators gives the differential equation

$$
\left(1-x^{2}\right) y^{\prime \prime}(x)-[\alpha-\beta+(\alpha+\beta+2) x] y^{\prime}(x)=-n(n+\alpha+\beta+1) y(x),
$$

where $y(x)=\hat{P}_{n}^{(\alpha, \beta)}(x)$, and repeated use of the raising operator $D_{\alpha, \beta}$ gives the Rodrigues formula

$$
(-1)^{n}\left(\begin{array}{c}
\alpha+\beta+2 n \\
n
\end{array}\right) n ! \hat{P}_{n}^{(\alpha, \beta)}(x)=(1-x)^{-\alpha}(1+x)^{-\beta} \frac{d^{n}}{d x^{n}}(1-x)^{n+\alpha}(1+x)^{n+\beta} .
$$

Using Leibnitz' formula we can obtain the explicit formula

$$
(-1)^{n}\left(\begin{array}{c}
\alpha+\beta+2 n \\
n
\end{array}\right) \hat{P}_{n}^{(\alpha, \beta)}(x)=\sum_{k=0}^{n}\left(\begin{array}{c}
n+\alpha \\
k
\end{array}\right)\left(\begin{array}{c}
n+\beta \\
n-k
\end{array}\right)(-1)^{k}(1-x)^{n-k}(1+x)^{k} .
$$

The historical way to normalize the Jacobi polynomials is by setting $P_{n}^{(\alpha, \beta)}(1)=$ $\left(\begin{array}{c}n+\alpha \\ n\end{array}\right)$ so that $P_{n}^{(\alpha, \beta)}(x)=2^{-n}\left(\begin{array}{c}\alpha+\beta+2 n \\ n\end{array}\right) \hat{P}_{n}^{(\alpha, \beta)}(x)$. See Szegö [Sz, Chapter IV] for more properties of the Jacobi polynomials.

1.2. Laguerre polynomials. The monic Laguerre polynomials $\hat{L}_{n}^{\alpha}$ satisfy the orthogonality conditions

$$
\int_{0}^{\infty} \hat{L}_{n}^{\alpha}(x) x^{\alpha} e^{-x} x^{k} d x=0, \quad k=0,1,2, \ldots, n-1,
$$

where $\alpha>-1$. Differentiation gives again the lowering operation

$$
D \hat{L}_{n}^{\alpha}(x)=n \hat{L}_{n-1}^{\alpha+1}(x),
$$

and $D_{\alpha}=x^{-\alpha} e^{x} D x^{\alpha} e^{-x}$ is the raising operator for which

$$
x D_{\alpha} \hat{L}_{n}^{\alpha}(x)=-\hat{L}_{n+1}^{\alpha-1}(x) .
$$


Both (1.14) and (1.15) can easily be proved using integration by parts and the orthogonality relation (1.13). Combining the lowering and the raising operator gives the differential equation

$$
x y^{\prime \prime}(x)+(\alpha+1-x) y^{\prime}(x)=-n y(x),
$$

where $y(x)=\hat{L}_{n}^{\alpha}(x)$. Using the raising operator repeatedly gives the Rodrigues formula

$$
(-1)^{n} \hat{L}_{n}^{\alpha}(x)=x^{-\alpha} e^{x} \frac{d^{n}}{d x^{n}} x^{\alpha+n} e^{-x}
$$

from which one can obtain an explicit expression using Leibnitz' formula

$$
(-1)^{n} \hat{L}_{n}^{\alpha}(x)=n ! \sum_{k=0}^{n}\left(\begin{array}{l}
n+\alpha \\
n-k
\end{array}\right) \frac{(-x)^{k}}{k !} .
$$

The historical normalization for the Laguerre polynomials is to take $L_{n}^{\alpha}(0)=\left(\begin{array}{c}n+\alpha \\ n\end{array}\right)$ so that $L_{n}^{\alpha}(x)=(-1)^{n} / n ! \hat{L}_{n}^{\alpha}(x)$. See Szegö [Sz, Chapter V] for more properties of Laguerre polynomials.

1.3. Hermite polynomials. The monic Hermite polynomials $\hat{H}_{n}$ satisfy the orthogonality relations

$$
\int_{-\infty}^{\infty} H_{n}(x) e^{-x^{2}} x^{k} d x=0, \quad k=0,1,2, \ldots, n-1 .
$$

Integration by parts and the orthogonality show that $D$ is a lowering operator

$$
D \hat{H}_{n}(x)=\hat{H}_{n}^{\prime}(x)=n \hat{H}_{n-1}(x)
$$

and we have the raising operator

$$
e^{x^{2}} D e^{-x^{2}} \hat{H}_{n}(x)=-2 \hat{H}_{n+1}(x) .
$$

Combining the lowering and the raising operator gives the differential equation

$$
y^{\prime \prime}(x)-2 x y^{\prime}(x)=-2 n y(x),
$$

where $y(x)=\hat{H}_{n}(x)$. Repeated application of the raising operator gives the Rodrigues formula

$$
(-1)^{n} 2^{n} \hat{H}_{n}(x)=e^{x^{2}} \frac{d^{n}}{d x^{n}} e^{-x^{2}},
$$

and by using Faa di Bruno's formula for the $n$th derivative of a composite function we obtain

$$
\hat{H}_{n}(x)=2^{-n} n ! \sum_{k=0}^{\lfloor n / 2\rfloor}(-1)^{k} \frac{(2 x)^{n-2 k}}{k !(n-2 k) !} .
$$

Historically the Hermite polynomials are normalized to have leading coefficient $2^{n}$, hence $H_{n}(x)=2^{n} \hat{H}_{n}(x)$. For more properties we refer to Szegö [Sz, Chapter 5]. 


\section{Multiple orthogonal polynomials}

During the past few years there has been increased interest in multiple orthogonal polynomials, particularly in Eastern Europe. See, e.g., surveys by Aptekarev [A2], de Bruin [dB] and Chapter 4 in the book of Nikishin and Sorokin [NS]. For multiple orthogonal polynomials we will need multi-indices consisting of $r$ positive integers, for which we use the notation $\vec{n}=\left(n_{1}, n_{2}, \ldots, n_{r}\right) \in \mathbb{N}^{r}$, where $r \in \mathbb{N}$. Furthermore we will use the notation $|\vec{n}|=n_{1}+n_{2}+\cdots+n_{r}$. We distribute the orthogonality conditions over $r$ real intervals $\Delta_{1}, \Delta_{2}, \ldots, \Delta_{r}$ with $r$ different measures $\mu_{1}, \mu_{2}, \ldots, \mu_{r}$. Two different ways are considered, which give type I and type II multiple orthogonal polynomials.

2.1. Type II multiple orthogonal polynomials. Type II multiple orthogonal polynomials are monic polynomials $P_{\vec{n}}$ of degree $|\vec{n}|$ such that

$$
\begin{aligned}
& \int_{\Delta_{1}} P_{\vec{n}}(x) x^{k} d \mu_{1}(x)=0, \quad k=0,1, \ldots, n_{1}-1, \\
& \int_{\Delta_{2}} P_{\vec{n}}(x) x^{k} d \mu_{2}(x)=0, \quad k=0,1, \ldots, n_{2}-1, \\
& \int_{\Delta_{r}} P_{\vec{n}}(x) x^{k} d \mu_{r}(x)=0, \quad k=0,1, \ldots, n_{r}-1 .
\end{aligned}
$$

For $r=1$ we get the ordinary monic orthogonal polynomials, but for $r>1$ we get multiple orthogonal polynomials (or polyorthogonal polynomials). All together the orthogonality conditions give $|\vec{n}|$ linear equations for the $|\vec{n}|$ unknown coefficients $a_{k, \vec{n}}$ of the polynomial $P_{\vec{n}}(x)=\sum_{k=0}^{|\vec{n}|} a_{k, \vec{n}} x^{k}$, where $a_{|\vec{n}|, \vec{n}}=1$. However, the matrix of coefficients of this system can be singular and we need some extra conditions on the $r$ measures $\mu_{1}, \mu_{2}, \ldots, \mu_{r}$ in order that the multiple orthogonal polynomial is unique. If the polynomial $P_{\vec{n}}(x)$ is unique, then we say that $\vec{n}$ is a normal index and if all indices are normal then we have a complete system. Our interest is of course in systems of $r$ measures for which all multi-indices are normal.

2.2. Type I multiple orthogonal polynomials. For type I multiple orthogonal polynomials we want to find a vector of polynomials $\left(A_{\vec{n}, 1}, A_{\vec{n}, 2}, \ldots, A_{\vec{n}, r}\right)$ such that each polynomial $A_{\vec{n}, j}$ is of degree $n_{j}-1$ and

$$
\sum_{j=1}^{r} \int_{\Delta_{j}} A_{\vec{n}, j}(x) x^{k} d \mu_{j}(x)=0, \quad k=0,1,2, \ldots,|\vec{n}|-2 .
$$

Again for $r=1$ we find the ordinary orthogonal polynomials, but for $r>1$ we find multiple orthogonal polynomials. There are $|\vec{n}|-1$ orthogonality conditions which give $|\vec{n}|-1$ homogenous linear equations for the $|\vec{n}|$ unknown coefficients of all the polynomials $A_{\vec{n}, j}(j=1,2, \ldots, r)$. Hence we can determine the vector $\left(A_{\vec{n}, 1}, A_{\vec{n}, 2}, \ldots, A_{\vec{n}, r}\right)$ up to a multiplicative factor.

2.3. Operator approach. The three-term recurrence relation (1.2) and the Jacobi matrix (1.6) have an interesting extension for multiple orthogonal polynomials, which was subsequently exploited by Kalyagin [K2] to initiate the spectral 
analysis of certain nonsymmetric operators. We construct a special sequence $\vec{s}(n)$ $\left(n \in \mathbb{N}\right.$ ) of multi-indices in $\mathbb{N}^{r}$ by writing $n=k r+j$, with $0 \leq j<r$ and then set

$$
\vec{s}(n)=(\underbrace{k+1, k+1, \ldots, k+1}_{j \text { times }}, k, k, \ldots, k) .
$$

These indices are nearly diagonal and if all these indices are normal, then we have a weakly complete system. Now consider the monic type II multiple orthogonal polynomials with these indices, i.e., we put $Q_{n}(x)=P_{\vec{s}(n)}(x)$, then one can use all the orthogonality relations $(2.1)-(2.3)$ to show that they satisfy a recurrence relation of the form

$$
x Q_{n}(x)=Q_{n+1}(x)+a_{0}(n) Q_{n}(x)+a_{1}(n) Q_{n-1}(x)+\cdots+a_{r}(n) Q_{n-r}(x),
$$

with initial conditions $Q_{0}=1$ and $Q_{-1}=Q_{-2}=\cdots=Q_{-r}=0$. These diagonal polynomials therefore satisfy a linear recurrence relation of order $r+1$, generalizing the second order recurrence relation for ordinary orthogonal polynomials $(r=1)$. We can then construct a banded Hessenberg matrix

$$
(2.7) H_{n}=\left(\begin{array}{ccccccc}
a_{0}(0) & 1 & & & & & 0 \\
a_{1}(1) & a_{0}(1) & 1 & & & & \\
\vdots & a_{1}(2) & a_{0}(2) & 1 & & & \\
a_{r}(r) & \vdots & a_{1}(3) & a_{0}(3) & 1 & & \\
& a_{r}(r+1) & \ddots & \ddots & \ddots & \ddots & 1 \\
& & \ddots & & & & a_{0}(n-1)
\end{array}\right) .
$$

Again it turns out that the eigenvalues of $H_{n}$ coincide with the zeros of the multiple orthogonal polynomial $Q_{n}=P_{\vec{s}(n)}$. The matrix $H_{n}$ is not symmetric, thus there is no a priori reason why the eigenvalues should be real. Nevertheless it turns out that in many cases the zeros are indeed real. Hence the coefficients $a_{i}(n+i)(n \in \mathbb{N})$, with $0 \leq i \leq r$ are not arbitrary and have some hidden structure. An interesting open problem is to find this hidden structure and to find out under which conditions on these coefficients the matrix $H_{n}$ corresponds to multiple orthogonal polynomials.

\section{Hermite-Padé approximation}

3.1. Simultaneous Padé approximation. Multiple orthogonal polynomials are closely related to simultaneous Padé approximation, which is nowadays known as Hermite-Padé approximation. Suppose we are given $r$ Markov functions (or Stieltjes functions)

$$
\begin{aligned}
& f_{1}(z)=\int_{\Delta_{1}} \frac{d \mu_{1}}{z-x}, \quad z \notin \Delta_{1}, \\
& f_{2}(z)=\int_{\Delta_{2}} \frac{d \mu_{2}}{z-x}, \quad z \notin \Delta_{2}, \\
& f_{r}(z)=\int_{\Delta_{r}} \frac{d \mu_{r}}{z-x}, \quad z \notin \Delta_{r},
\end{aligned}
$$


then our aim is to approximate these $r$ functions simultaneously by rational functions, such that the approximation is of prescribed order near $\infty$. Type I HermitePadé approximation is to find a vector of polynomials $\left(A_{\vec{n}, 1}, A_{\vec{n}, 2}, \ldots, A_{\vec{n}, r}\right)$ and a polynomial $B_{\vec{n}}$ such that the degree of $A_{\vec{n}, j}$ is less than or equal to $n_{j}-1$ and

$$
A_{\vec{n}, 1}(z) f_{1}(z)+\cdots+A_{\vec{n}, r}(z) f_{r}(z)-B_{\vec{n}}(z)=\mathcal{O}\left(\frac{1}{z^{|\vec{n}|}}\right), \quad z \rightarrow \infty
$$

Type II Hermite-Padé approximation is to find a polynomial $P_{\vec{n}}$ of degree $|\vec{n}|$ and polynomials $Q_{\vec{n}, 1}, \ldots, Q_{\vec{n}, r}$ such that

$$
\begin{aligned}
& P_{\vec{n}}(z) f_{1}(z)-Q_{\vec{n}, 1}(z)=\mathcal{O}\left(\frac{1}{z^{n_{1}+1}}\right), z \rightarrow \infty \\
& P_{\vec{n}}(z) f_{2}(z)-Q_{\vec{n}, 2}(z)=\mathcal{O}\left(\frac{1}{z^{n_{2}+1}}\right), \quad z \rightarrow \infty \\
& \vdots \\
& P_{\vec{n}}(z) f_{r}(z)-Q_{\vec{n}, r}(z)=\mathcal{O}\left(\frac{1}{z^{n_{r}+1}}\right), \quad z \rightarrow \infty
\end{aligned}
$$

Hence type II Hermite-Padé approximation is rational approximation of the functions $f_{1}, f_{2}, \ldots, f_{r}$ with a common denominator. For $r=1$ type $\mathrm{I}$ and type II Hermite-Padé approximation reduces to ordinary Padé approximation of $f_{1}$. It is well known that the denominator polynomials in Padé approximation to a Markov function (or Stieltjes function) are orthogonal polynomials for the measure $\mu_{1}$. For multiple orthogonal polynomials there is a similar relationship: the polynomials $A_{\vec{n}, 1}, A_{\vec{n}, 2}, \ldots, A_{\vec{n}, r}$ in type I Hermite-Padé approximation are precisely the type I multiple orthogonal polynomials for the measures $\left(\mu_{1}, \ldots, \mu_{r}\right)$ and the common denominator $P_{\vec{n}}$ in type II Hermite-Padé approximation is the type II multiple orthogonal polynomial for the measures $\left(\mu_{1}, \ldots, \mu_{r}\right)$. Furthermore, the remaining polynomials $B_{\vec{n}}$ in type I approximation and $Q_{\vec{n}, j}(j=1,2, \ldots, r)$ in type II approximation can be obtained from the multiple orthogonal polynomials through the formulas

$$
B_{\vec{n}}(z)=\sum_{j=1}^{r} \int_{\Delta_{j}} \frac{A_{\vec{n}, j}(z)-A_{\vec{n}, j}(x)}{z-x} d \mu_{j}(x),
$$

and

$$
Q_{\vec{n}, j}(z)=\int_{\Delta_{j}} \frac{P_{\vec{n}}(z)-P_{\vec{n}}(x)}{z-x} d \mu_{j}(x), \quad j=1, \ldots, r .
$$

Combining (3.1) with (3.5) gives a nice formula for the remainder in type I HermitePadé approximation in terms of the multiple orthogonal polynomials

$$
\sum_{j=1}^{r} A_{\vec{n}, j}(z) f_{j}(z)-B_{\vec{n}}(z)=\sum_{j=1}^{r} \int_{\Delta_{j}} \frac{A_{\vec{n}, j}(x)}{z-x} d \mu_{j}(x),
$$

and combining (3.2)-(3.4) with (3.6) gives a formula for the remainder in type II Hermite-Padé approximation

$$
P_{\vec{n}}(z) f_{j}(z)-Q_{\vec{n}, j}(z)=\int_{\Delta_{j}} \frac{P_{\vec{n}}(x)}{z-x} d \mu_{j}(x) .
$$


Hence for Hermite-Padé approximation one only needs to obtain the multiple orthogonal polynomials, from which all other quantities can be deduced.

3.2. Useful systems of functions. In order to get a system for which most multi-indices are normal, one needs to pose additional assumptions on the Markov functions $\left(f_{1}, f_{2}, \ldots, f_{r}\right)$ or on the measures $\left(\mu_{1}, \mu_{2}, \ldots, \mu_{r}\right)$. Two kinds of systems turn out to be quite useful.

Definition 3.1 (see [An]). An Angelesco system consists of $r$ measures on $r$ pairwise disjoint intervals:

$$
f_{j}(z)=\int_{\Delta_{j}} \frac{d \mu_{j}(x)}{z-x}, \quad \Delta_{i} \cap \Delta_{j}=\emptyset \text { if } i \neq j .
$$

Angelesco systems are always complete systems and are the prototype for studying multiple orthogonal polynomials on $r$ pairwise disjoint intervals. It turns out that the zeros of type II multiple orthogonal polynomials have a very simple location property for Angelesco systems: there will be $n_{i}$ zeros of $P_{\vec{n}}$ on the interval $\Delta_{i}$ for $i=1,2, \ldots, r$. The polynomial $P_{\vec{n}}$ can therefore be factored as $P_{\vec{n}}(x)=P_{n_{1}}(x) P_{n_{2}}(x) \cdots P_{n_{r}}(x)$ and each $P_{n_{i}}$ is an ordinary orthogonal polynomial on $\Delta_{i}$ for the varying measure $\prod_{j \neq i} P_{n_{j}}(x) d \mu_{i}(x)$.

For multiple orthogonal polynomials on one interval there is another useful system of functions.

Definition 3.2 (see [N1]). An AT system consists of $r$ measures on the same interval $\Delta$ such that each measure $\mu_{j}$ is absolutely continuous with respect to $\mu=\mu_{1}$ and such that the Radon-Nikodym derivatives $u_{j}=d \mu_{j} / d \mu\left(\right.$ with $\left.u_{1}=1\right)$ are such that $\left\{1, x, \ldots, x^{n_{1}-1}, u_{2}, x u_{2}, \ldots, x^{n_{2}-1} u_{2}, \ldots, u_{r}, x u_{r}, \ldots, x^{n_{r}-1} u_{r}\right\}$ is a Chebyshev system on $\Delta$ for every multi-index $\left(n_{1}, n_{2}, \ldots, n_{r}\right)$.

Recall that a set of $n$ linearly independent functions $\left\{\phi_{1}, \phi_{2}, \ldots, \phi_{n}\right\}$ is a Chebyshev system on $\Delta$ if every linear combination $\sum_{k=1}^{n} a_{k} \phi_{k}$ has at most $n-1$ zeros on $\Delta$. For an AT system it thus follows that $P_{n_{1}-1}(x)+P_{n_{2}-1}(x) u_{2}(x)+$ $\cdots+P_{n_{r}-1}(x) u_{r}(x)$ has at most $|\vec{n}|-1$ zeros on $\Delta$ whenever $P_{k}$ is a polynomial of degree at most $k$. A special example of an AT system is a Nikishin system [N2] which is defined recursively as follows. Any Markov function

$$
f_{1,1}(z)=\int_{\Delta_{1}} \frac{d \mu_{1}(x)}{z-x}
$$

is a Nikishin system of order one on $\Delta_{1}$. A system $\left(f_{2,1}, f_{2,2}\right)$ is a Nikishin system of order two on $\Delta_{2}$ if

$$
f_{2,1}(z)=\int_{\Delta_{2}} \frac{d \mu_{2}(x)}{z-x}, \quad f_{2,2}(z)=\int_{\Delta_{2}} f_{1,1}(x) \frac{d \mu_{2}(x)}{z-x}
$$

where $f_{1,1}$ is a Nikishin system of order one on $\Delta_{1}$ and $\Delta_{1} \cap \Delta_{2}=\emptyset$. In general the system $\left(f_{r, 1}, f_{r, 2}, \ldots, f_{r, r}\right)$ is a Nikishin system of order $r$ on $\Delta_{r}$ if

$$
f_{r, j}(z)=\int_{\Delta_{r}} f_{r-1, j-1}(x) \frac{d \mu_{r}(x)}{z-x}, \quad j=1,2, \ldots, r
$$

where $f_{r-1,0}=1$ and $\left(f_{r-1,1}, f_{r-1,2}, \ldots, f_{r-1, r-1}\right)$ is a Nikishin system of order $r-1$ on $\Delta_{r-1}$ with $\Delta_{r} \cap \Delta_{r-1}=\emptyset$. Nikishin systems turn out to be the prototype of systems of Markov functions on one interval and allow a rather detailed analysis. See, e.g., Driver and Stahl [DS1] [DS2] for normality of Nikishin systems and 
Bustamante and López [BL] and Aptekarev [A3] for asymptotic results. Driver and Stahl [DS1] have shown that the type II multiple orthogonal polynomial $P_{\vec{n}}$ for a Nikishin system of order $r$ on $\Delta_{r}$ has $|\vec{n}|$ simple zeros on this interval $\Delta_{r}$ whenever $n_{j} \geq \max \left(n_{j+1}, \ldots, n_{r}\right)-1$ for every $j=1,2, \ldots, r-1$. In particular this is true for the diagonal indices $\vec{s}(n)$ given in $(2.5)$.

Recently Gonchar, Rakhmanov and Sorokin [GRS] have considered a mixture of Angelesco and Nikishin systems and have analyzed the asymptotic behavior of the corresponding Hermite-Padé approximants. The asymptotic behavior of the Hermite-Padé approximants for Angelesco and Nikishin systems was obtained in [GR] and [S5]. One of the important results is the asymptotic distribution of the zeros of the polynomials. For ordinary orthogonal polynomials the distribution of zeros is usually obtained by investigating an extremal problem for logarithmic potentials. For multiple orthogonal polynomials one needs to study an extremal problem in logarithmic potential theory for vector potentials.

\section{Examples of multiple orthogonal polynomials}

The very classical orthogonal polynomials of Jacobi, Laguerre, and Hermite have several possible extensions as multiple orthogonal polynomials. We will mention some of them. See also Aptekarev, Marcellán and Rocha [AMR] for some Angelesco type multiple orthogonal polynomials.

4.1. Jacobi-Angelesco polynomials. Kalyagin $[\mathbf{K 1}][\mathbf{K R}]$ studied multiple Jacobi polynomials on two intervals $[a, 0]$ and $[0,1]$, with $a<0$. The measures are $d \mu_{1}(x)=|x|^{\gamma}(x-a)^{\alpha}(1-x)^{\beta} d x$ on $[a, 0]$ and $d \mu_{2}(x)=x^{\gamma}(x-a)^{\alpha}(1-x)^{\beta} d x$ on $[0,1]$, with $\alpha, \beta, \gamma>-1$. These intervals have only one point in common and this point will not influence the nature of the multiple orthogonal polynomials so that we can still consider this as an Angelesco system. There is a raising operator for the monic diagonal type II multiple orthogonal polynomials, namely

$$
\begin{aligned}
& \left(x^{\gamma}(x-a)^{\alpha}(1-x)^{\beta} P_{n, n}^{(\alpha, \beta, \gamma)}(x)\right)^{\prime} \\
& \quad=-(\alpha+\beta+\gamma+2 n) x^{\gamma-1}(x-a)^{\alpha-1}(1-x)^{\beta-1} P_{n+1, n+1}^{(\alpha-1, \beta-1, \gamma-1)}(x),
\end{aligned}
$$

and by using this formula repeatedly we obtain the Rodrigues formula

$$
\begin{aligned}
& (-1)^{n} n !\left(\begin{array}{c}
\alpha+\beta+\gamma+3 n \\
n
\end{array}\right) P_{n, n}^{(\alpha, \beta, \gamma)}(x) \\
& \quad=x^{-\gamma}(x-a)^{-\alpha}(1-x)^{-\beta} \frac{d^{n}}{d x^{n}}\left(x^{\gamma+n}(x-a)^{\alpha+n}(1-x)^{\beta+n}\right)
\end{aligned}
$$

These polynomials satisfy a third order differential equation

$$
\begin{aligned}
x(x-1)(x-a) & y^{\prime \prime \prime}+\left(6 x^{2}-4(a+1) x+2 a\right) y^{\prime \prime} \\
& +(n-1)(n+2)(-3 x+a+1) y^{\prime}-2 n\left(n^{2}+3 n+2\right) y=0,
\end{aligned}
$$

where $y(x)=P_{n, n}^{(\alpha, \beta, \gamma)}(x)$. Observe that the coefficients of $y^{\prime \prime \prime}$ and $y^{\prime \prime}$ are polynomials independent of $n$ but that the coefficient of $y^{\prime}$ depends on $n$, so that SturmLiouville theory of linear differential equations can not be used here. If we write $P_{2 n}=P_{n, n}^{(\alpha, \beta, \gamma)}$ and $P_{2 n+1}=P_{n+1, n}^{(\alpha, \beta, \gamma)}$, then we have a third order recurrence relation

$$
x P_{n}(x)=P_{n+1}(x)+b_{n} P_{n}(x)+c_{n} P_{n-1}(x)+d_{n} P_{n-2}(x),
$$


for which

$$
\begin{array}{cc}
\lim _{n \rightarrow \infty} b_{2 n}=\frac{a+1}{9}+\frac{2}{3} x_{1}, & \lim _{n \rightarrow \infty} b_{2 n+1}=\frac{a+1}{9}+\frac{2}{3} x_{2}, \\
\lim _{n \rightarrow \infty} c_{n}=\frac{4}{81}\left(a^{2}-a+1\right), & \\
\lim _{n \rightarrow \infty} d_{2 n}=-\frac{4}{27} x_{1}\left(x_{1}-a\right)\left(x_{1}-1\right), & \lim _{n \rightarrow \infty} d_{2 n+1}=-\frac{4}{27} x_{2}\left(x_{2}-a\right)\left(x_{2}-1\right)
\end{array}
$$

where $x_{1}$ and $x_{2}$ are the roots of the quadratic equation $h^{\prime}(x)=0$, with $h(x)=$ $x(x-a)(x-1)$.

4.2. Jacobi-Pineiro polynomials. Pineiro $[\mathbf{P}]$ considered multiple Jacobi polynomials on $[0,1]$ with Jacobi weights

$$
d \mu_{j}(x)=x^{\alpha_{j}}(1-x)^{\alpha_{0}} d x, \quad j=1,2, \ldots, r,
$$

where $\alpha_{k}>-1$ for $k=0,1, \ldots, r$ and $\alpha_{i}-\alpha_{j} \notin \mathbb{Z}$ whenever $i \neq j$ and $i, j \neq 0$. Originally Pineiro only considered the case $\alpha_{0}=0$ but this more general case can easily be obtained without too much extra effort (see, e.g., [NS, p. 162]). If we set $\vec{\alpha}=\left(\alpha_{1}, \ldots, \alpha_{r}\right)$ then we have $r$ raising operators for the monic type II multiple orthogonal polynomials:

$$
\begin{aligned}
& \left(x^{\alpha_{j}}(1-x)^{\alpha_{0}} P_{\vec{n}}^{\alpha_{0}, \vec{\alpha}}(x)\right)^{\prime} \\
& \quad=-\left(|\vec{n}|+\alpha_{0}+\alpha_{j}\right) x^{\alpha_{j}-1}(1-x)^{\alpha_{0}-1} P_{\vec{n}+\vec{e}_{j}}^{\alpha_{0}-1, \vec{\alpha}-\vec{e}_{j}}(x), \quad j=1, \ldots, r,
\end{aligned}
$$

where $\vec{e}_{j}$ is the $j$ th standard unit vector $(0, \ldots, 0,1,0, \ldots, 0)$. Combining all these raising operators gives the Rodrigues formula

$$
\begin{aligned}
& (-1)^{|\vec{n}|} \prod_{j=1}^{r}\left(|\vec{n}|+\alpha_{0}+\alpha_{j}+1\right)_{n_{j}} P_{\vec{n}}^{\alpha_{0}, \vec{\alpha}}(x) \\
& \quad=(1-x)^{-\alpha_{0}} \prod_{j=1}^{r}\left[x^{-\alpha_{j}} \frac{d^{n_{j}}}{d x^{n_{j}}} x^{n_{j}+\alpha_{j}}\right](1-x)^{\alpha_{0}+|\vec{n}|}
\end{aligned}
$$

where the order in the product of $r$ differential operators can be changed. For $r=2$ we put $P_{2 n}=P_{n, n}^{\left(\alpha_{0}, \alpha_{1}, \alpha_{2}\right)}$ and $P_{2 n+1}=P_{n+1, n}^{\left(\alpha_{0}, \alpha_{1}, \alpha_{2}\right)}$ and we have the third order recurrence relation

$$
x P_{n}(x)=P_{n+1}(x)+b_{n} P_{n}(x)+c_{n} P_{n-1}(x)+d_{n} P_{n-2}(x),
$$

for which the recurrence coefficients satisfy

$$
\begin{aligned}
\lim _{n \rightarrow \infty} b_{n} & =3 \frac{4}{27}, \\
\lim _{n \rightarrow \infty} c_{n} & =3\left(\frac{4}{27}\right)^{2}, \\
\lim _{n \rightarrow \infty} d_{n} & =\left(\frac{4}{27}\right)^{3} .
\end{aligned}
$$


4.3. Multiple Laguerre polynomials. In a similar way, Sorokin [S2] [S6] considered multiple Laguerre polynomials on $[0, \infty)$ with weights

$$
d \mu_{j}(x)=x^{\alpha_{j}} e^{-x} d x, \quad j=1,2, \ldots, r
$$

with $\alpha_{j}>-1$ for $j=1,2, \ldots, r$ and $\alpha_{i}-\alpha_{j} \notin \mathbb{Z}$ for $i \neq j$. These polynomials are a limiting case of the Jacobi-Pineiro polynomials:

$$
\lim _{\alpha_{0} \rightarrow \infty} \alpha_{0}^{|\vec{n}|} P_{\vec{n}}^{\alpha_{0}, \vec{\alpha}}\left(x / \alpha_{0}\right)=L_{\vec{n}}^{\vec{\alpha}}(x),
$$

in a similar way as the Laguerre polynomials are a limiting case of the Jacobi polynomials. There are again $r$ raising operators

$$
\left(x^{\alpha_{j}} e^{-x} L_{\vec{n}}^{\vec{\alpha}}(x)\right)^{\prime}=-x^{\alpha_{j}-1} e^{-x} L_{\vec{n}+\vec{e}_{j}}^{\vec{\alpha}-\vec{e}_{j}}(x), \quad j=1,2 \ldots, r
$$

and combining these raising operators gives the Rodrigues formula

$$
(-1)^{|\vec{n}|} L_{\vec{n}}^{\vec{\alpha}}(x)=e^{x} \prod_{j=1}^{r}\left[x^{-\alpha_{j}} \frac{d^{n_{j}}}{d x^{n_{j}}} x^{n_{j}+\alpha_{j}}\right] e^{-x} .
$$

For $r=2$ we put $P_{2 n}=L_{n, n}^{\left(\alpha_{1}, \alpha_{2}\right)}$ and $P_{2 n+1}=L_{n+1, n}^{\left(\alpha_{1}, \alpha_{2}\right)}$ to find the third order recurrence relation

$$
x P_{n}(x)=P_{n+1}(x)+b_{n} P_{n}(x)+c_{n} P_{n-1}(x)+d_{n} P_{n-2}(x),
$$

with

$$
\begin{aligned}
b_{2 n} & =3 n+\alpha_{1}+1 \\
b_{2 n+1} & =3 n+\alpha_{2}+2 \\
c_{2 n} & =n\left(3 n+\alpha_{1}+\alpha_{2}\right) \\
c_{2 n+1} & =(n+1)\left(3 n+\alpha_{1}+\alpha_{2}\right)-\alpha_{2}+1 \\
d_{2 n} & =n\left(n+\alpha_{1}\right)\left(n+\alpha_{1}-\alpha_{2}\right) \\
d_{2 n+1} & =n\left(n+\alpha_{2}\right)\left(n+\alpha_{2}-\alpha_{1}\right) .
\end{aligned}
$$

Observe that

$$
b_{n}=3 \frac{n}{2}+\mathcal{O}(1), \quad c_{n}=3\left(\frac{n}{2}\right)^{2}+\mathcal{O}(n), \quad d_{n}=\left(\frac{n}{2}\right)^{3}+\mathcal{O}\left(n^{2}\right) .
$$

Another way to obtain multiple Laguerre polynomials on $[0, \infty)$ is to use the weights

$$
d \mu_{j}(x)=x^{\alpha} e^{-c_{j} x} d x, \quad j=1,2, \ldots, r,
$$

with $\alpha>-1$ and $c_{1}, c_{2}, \ldots, c_{r}$ distinct positive real numbers (see [NS, p. 160] where $\alpha=0$ ). The raising operators are

$$
\left(x^{\alpha} e^{-c_{j} x} P_{\vec{n}}^{\vec{c}, \alpha}(x)\right)^{\prime}=-c_{j} x^{\alpha-1} e^{-c_{j} x} P_{\vec{n}+\vec{e}_{j}}^{\vec{c}, \alpha-1}(x), \quad j=1,2, \ldots, r,
$$

and the corresponding Rodrigues formula is

$$
(-1)^{|\vec{n}|} \prod_{j=1}^{r} c_{j}^{n_{j}} P_{\vec{n}}^{\vec{c}, \alpha}(x)=x^{-\alpha} \prod_{j=1}^{r}\left[e^{c_{j} x} \frac{d^{n_{j}}}{d x^{n_{j}}} e^{-c_{j} x}\right] x^{|\vec{n}|+\alpha},
$$

and again the product of the differential operators can be taken in any order. 
4.4. Jacobi-Laguerre polynomials. Sorokin [S1] considered an Angelesco case containing a Jacobi and a Laguerre part. Consider the measures $d \mu_{1}(x)=(1+$ $x)^{\alpha}|x|^{\beta} e^{-x} d x$ on the interval $[-1,0]$ and $d \mu_{2}(x)=(1+x)^{\alpha} x^{\beta} e^{-x} d x$ on $[0, \infty)$, with $\alpha, \beta>-1$, then the raising operator for the diagonal type II multiple orthogonal polynomial is

$$
\left((1+x)^{\alpha} x^{\beta} e^{-x} P_{n, n}^{(\alpha, \beta)}(x)\right)^{\prime}=-(1+x)^{\alpha-1} x^{\beta-1} e^{-x} P_{n+1, n+1}^{(\alpha-1, \beta-1)}(x),
$$

and applying this rule repeatedly gives the Rodrigues formula

$$
(-1)^{n} P_{n, n}^{(\alpha, \beta)}(x)=(1+x)^{-\alpha} x^{-\beta} e^{x} \frac{d^{n}}{d x^{n}}(1+x)^{n+\alpha} x^{n+\beta} e^{-x} .
$$

4.5. Multiple Macdonald polynomials. For $r=1$ the very classical orthogonal polynomials are those of Jacobi, Laguerre and Hermite. For multiple orthogonal polynomials there seem to be more classical families, which is at least suggested by the following example [VAY]. Consider the weight function

$$
d \mu_{1}(x)=x^{\alpha} \rho_{\nu}(x) d x, \quad d \mu_{2}(x)=x^{\alpha} \rho_{\nu+1}(x) d x \quad x \in[0, \infty),
$$

where $\alpha>-1$ and $\rho_{\nu}(x)=x^{\nu / 2} K_{\nu}(2 \sqrt{x})(\nu \geq 0)$, with $K_{\nu}$ the Macdonald function (modified Bessel function of the second kind). This AT system has a lot of properties that very classical polynomials usually have. The differential operator $D$ is a lowering operator for the type II multiple orthogonal polynomials near the diagonal

$$
D P_{n, n}^{\alpha}(x)=2 n P_{n, n-1}^{\alpha+1}(x), \quad D P_{n, n-1}^{\alpha}(x)=(2 n-1) P_{n-1, n-1}^{\alpha+1}(x),
$$

and $D_{\alpha}=x^{-\alpha} D x^{\alpha}$ is a raising operator for the type I multiple orthogonal polynomials: putting $Q_{m, n}^{\alpha}=A_{(m, n), 1}^{\alpha} \rho_{\nu}+A_{(m, n), 2}^{\alpha} \rho_{\nu+1}$ we have

$$
x D_{\alpha} Q_{n, n}^{\alpha}(x)=Q_{n+1, n}^{\alpha-1}(x), \quad x D_{\alpha} Q_{n, n-1}^{\alpha}(x)=Q_{n, n}^{\alpha-1}(x) .
$$

Using the raising operators repeatedly gives a Rodrigues formula for the type I multiple orthogonal polynomials

$$
x^{\alpha} Q_{n, n-1}^{\alpha}(x)=\frac{d^{2 n}}{x^{2 n}} x^{2 n+\alpha} \rho_{\nu}(x), \quad x^{\alpha} Q_{n, n}^{\alpha}(x)=\frac{d^{2 n+1}}{x^{2 n+1}} x^{2 n+1+\alpha} \rho_{\nu}(x) .
$$

Putting $P_{2 n}=P_{n, n}^{\alpha}$ and $P_{2 n+1}=P_{n+1, n}^{\alpha}$ gives the recurrence relation

$$
x P_{n}(x)=P_{n+1}(x)+b_{n} P_{n}(x)+c_{n} P_{n-1}(x)+d_{n} P_{n-2}(x),
$$

with

$$
\begin{aligned}
b_{n} & =(n+\alpha+1)(3 n+\alpha+2 \nu)-(\alpha+1)(\nu-1), \\
c_{n} & =n(n+\alpha)(n+\alpha+\nu)(3 n+2 \alpha+\nu), \\
d_{n} & =n(n-1)(n+\alpha-1)(n+\alpha)(n+\alpha+\nu-1)(n+\alpha+\nu)
\end{aligned}
$$

so that

$$
b_{n}=3 n^{2}+\mathcal{O}(n), \quad c_{n}=3 n^{4}+\mathcal{O}\left(n^{3}\right), \quad d_{n}=n^{6}+\mathcal{O}\left(n^{5}\right)
$$




\section{Irrationality}

Multiple orthogonal polynomials turn out to be quite useful in proving irrationality of certain real numbers. The key to such irrationality proofs is the following lemma:

Lemma 5.1. Let $x$ be a real number. Suppose there exists integers $p_{n}$ and $q_{n}$ $(n \in \mathbb{N})$ such that

1. $q_{n} x-p_{n} \neq 0$ for all $n \in \mathbb{N}$,

2. $\lim _{n \rightarrow \infty}\left(q_{n} x-p_{n}\right)=0$.

Then $x$ is irrational.

Proof. Suppose $x$ is rational so that $x=p / q$ with integers $p$ and $q$. Then

$$
q_{n} x-p_{n}=\frac{q_{n} p-p_{n} q}{q}
$$

By assumption (1) we know that $q_{n} p-p_{n} q$ is an integer different from zero, hence $\left|q_{n} p-p_{n} q\right| \geq 1$. But then

$$
\left|q_{n} x-p_{n}\right| \geq \frac{1}{q}
$$

and as $n \rightarrow \infty$ we find that this is in contradiction with assumption (2). Hence $x$ cannot be rational.

This lemma shows that if one can find good rational approximants $p_{n} / q_{n}$ such that $\left|x-p_{n} / q_{n}\right|=o\left(1 / q_{n}\right)$, then $x$ has to be irrational. Such irrational numbers can therefore be approximated very well by rational numbers. Constructing rational approximants to $x$ can be done using Padé approximation or Hermite-Padé approximation, especially when $x$ is the value of a Markov function $f(z)$ with $z$ far enough away from the support of the measure. This is the case for some values of the zeta function

$$
\zeta(s)=\sum_{n=1}^{\infty} \frac{1}{n^{s}}, \quad s>1
$$

in particular we have for all positive integers

$$
\zeta(j+1)=\frac{(-1)^{j}}{j !} \int_{0}^{1} \frac{\log ^{j} x}{1-x} d x .
$$

We can use that information and multiple orthogonal polynomials for the measures $d \mu_{j}(x)=(-1)^{j} \log ^{j}(x) d x$ on $[0,1]$ for $j=0,1,2$ to prove the irrationality of $\zeta(3)$. When Apéry [Ap] first announced the proof of irrationality of $\zeta(3)$, the proof was considered as rather mysterious. Later Beukers $[\mathbf{B 1}][\mathbf{B 2}]$ showed how Apéry's approximants can be obtained using Hermite-Padé approximation.

Theorem 5.2 (Apéry). $\zeta(3)$ is irrational.

Proof. In order to obtain good rational approximants to $\zeta(3)$ we will use Hermite-Padé approximation to the three Markov functions

$$
f_{1}(z)=\int_{0}^{1} \frac{1}{z-x} d x, \quad f_{2}(z)=-\int_{0}^{1} \frac{\log x}{z-x} d x, \quad f_{3}(z)=\frac{1}{2} \int_{0}^{1} \frac{\log ^{2} x}{z-x} d x
$$


and use that $f_{3}(1)=\zeta(3)$. We want to find a vector of polynomials $\left(A_{n}, B_{n}\right)$, where $A_{n}$ and $B_{n}$ are polynomials of degree $n$, and polynomials $C_{n}$ and $D_{n}$ such that

$$
\begin{aligned}
A_{n}(1) & =0 \\
A_{n}(z) f_{1}(z)+B_{n}(z) f_{2}(z)-C_{n}(z) & =\mathcal{O}\left(1 / z^{n+1}\right), \\
A_{n}(z) f_{2}(z)+2 B_{n}(z) f_{3}(z)-D_{n}(z) & =\mathcal{O}\left(1 / z^{n+1}\right) .
\end{aligned}
$$

Observe that (5.2) is a type I approximation problem for the system $\left(f_{1}, f_{2}\right)$ and (5.3) is a type $\mathbf{I}$ approximation problem for $\left(f_{2}, f_{3}\right)$, whereas the combination (5.2)(5.3) is a vector type II approximation problem with common vector denominator $\left(A_{n}, B_{n}\right)$. The solution of this problem in Hermite-Padé approximation is given by the generalized polynomial $A_{n}(x)-B_{n}(x) \log x$ for which the following orthogonality relations hold:

$$
\begin{aligned}
\int_{0}^{1}\left(A_{n}(x)-B_{n}(x) \log x\right) x^{k} d x & =0, \quad k=0,1, \ldots, n-1 \\
\int_{0}^{1}\left(A_{n}(x)-B_{n}(x) \log x\right) \log (x) x^{k} d x & =0, \quad k=0,1, \ldots, n-1,
\end{aligned}
$$

together with the extra condition that $A_{n}(1)=0$. Let $P_{n}$ be the Legendre polynomials of degree $n$ on $[0,1]$,

$$
P_{n}(x)=\frac{1}{n !} \frac{d^{n}}{d x^{n}} x^{n}(1-x)^{n}=\sum_{k=0}^{n}\left(\begin{array}{l}
n \\
k
\end{array}\right)\left(\begin{array}{c}
n+k \\
k
\end{array}\right)(-1)^{k} x^{k},
$$

then the Mellin convolution

$$
F_{n}(x)=\int_{x}^{1} P_{n}(x / t) P_{n}(t) \frac{d t}{t}
$$

is of the form $A_{n}(x)-B_{n}(x) \log x$ with $A_{n}(1)=0$. Taking the Mellin transform gives

$$
\int_{0}^{1} F_{n}(x) x^{s} d x=\left(\int_{0}^{1} P_{n}(x) x^{s} d x\right)^{2},
$$

and for $s=0,1, \ldots, n-1$ this gives the orthogonality conditions in (5.4). Taking derivatives gives

$$
\int_{0}^{1} F_{n}(x) x^{s} \log x d x=2 \int_{0}^{1} P_{n}(x) x^{s} d x \int_{0}^{1} P_{n}(x) x^{s} \log x d x,
$$

and for $s=0,1, \ldots, n-1$ this gives the orthogonality conditions (5.5). The remainder in (5.3) is

$$
-\int_{0}^{1} \frac{A_{n}(x)-B_{n}(x) \log x}{z-x} \log x d x,
$$

and hence, choosing $z=1$ in (5.3) gives

$$
2 B_{n}(1) \zeta(3)-D_{n}(1)=-\int_{0}^{1} \frac{A_{n}(x)-B_{n}(x) \log x}{1-x} \log x d x .
$$

Using the representation (5.7), the Rodrigues formula for $P_{n}$ and

$$
-\frac{\log x}{1-x}=\int_{0}^{1} \frac{d z}{1-(1-x) z}
$$


gives

$$
\begin{aligned}
&-\int_{0}^{1} \frac{A_{n}(x)-B_{n}(x) \log x}{1-x} \log x d x \\
&=\int_{0}^{1} \int_{0}^{1} \int_{0}^{1} \frac{x^{n} y^{n} z^{n}(1-x)^{n}(1-y)^{n}(1-z)^{n}}{(1-(1-x y) z)^{n+1}} d x d y d z>0,
\end{aligned}
$$

so that $2 B_{n}(1) \zeta(3)-D_{n}(1)>0$. A simple estimation of $x y z(1-x)(1-y)(1-$ $z) /(1-(1-x y) z)$ on the unit cube gives the upper bound

$$
2 B_{n}(1) \zeta(3)-D_{n}(1) \leq 2 \zeta(3)(\sqrt{2}-1)^{4 n} .
$$

Explicit calculation gives

$$
B_{n}(1)=\sum_{k=0}^{n}\left(\begin{array}{l}
n \\
k
\end{array}\right)^{2}\left(\begin{array}{c}
n+k \\
k
\end{array}\right)^{2}
$$

and

$$
\begin{aligned}
D_{n}(1)= & -\int_{0}^{1} \frac{A_{n}(1)-A_{n}(x)}{1-x} \log (x) d x+\int_{0}^{1} \frac{B_{n}(1)-B_{n}(x)}{1-x} \log ^{2} x d x \\
= & \sum_{k=0}^{n}\left(\begin{array}{c}
n \\
k
\end{array}\right)^{2}\left(\begin{array}{c}
n+k \\
k
\end{array}\right)^{2} \sum_{j=0}^{k-1} \frac{2}{(j+1)^{3}} \\
& +\sum_{k=0}^{n} \sum_{\substack{j=0 \\
j \neq k}}^{n}\left(\begin{array}{l}
n \\
k
\end{array}\right)\left(\begin{array}{c}
n \\
j
\end{array}\right)\left(\begin{array}{c}
n+k \\
k
\end{array}\right)\left(\begin{array}{c}
n+j \\
j
\end{array}\right)(-1)^{k+j} \frac{H_{k}^{(2)}-H_{j}^{(2)}}{j-k},
\end{aligned}
$$

where $H_{k}^{(2)}=\sum_{j=1}^{k} 1 / j^{2}$. Clearly $B_{n}(1)$ is a positive integer, but $D_{n}(1)$ contains rational terms. However, if we multiply $D_{n}(1)$ by a common multiple of the numbers $2^{3}, 3^{3}, \ldots, n^{3}$ then we would get an integer. Taking $d_{n}=\operatorname{lcm}(1,2,3, \ldots, n)$ (least common multiple) then

$$
0<2 d_{n}^{3} B_{n}(1) \zeta(3)-d_{n}^{3} D_{n}(1) \leq d_{n}^{3} \zeta(3)(\sqrt{2}-1)^{4 n} .
$$

Taking the $n$th root and using the asymptotic property

$$
\lim _{n \rightarrow \infty} d_{n}^{1 / n}=e,
$$

then gives, with $p_{n}=2 d_{n}^{3} B_{n}(1) \in \mathbb{N}$ and $q_{n}=d_{n}^{3} D_{n}(1) \in \mathbb{Z}$

$$
\limsup _{n \rightarrow \infty}\left(p_{n} \zeta(3)-q_{n}\right)^{1 / n} \leq e^{3}(\sqrt{2}-1)^{4}<1
$$

hence Lemma 5.1 implies that $\zeta(3)$ is irrational.

\section{Transcendence}

Historically, Hermite introduced the notion of Hermite-Padé approximation to prove the transcendence of $e$. The basis lemma for proving transcendence is a generalization of Lemma 5.1, which goes as follows:

Lemma 6.1. Let $x$ be a real number. Suppose that for every positive integer $m \in$ $\mathbb{N}$ and for all integers $a_{0}, a_{1}, \ldots, a_{m} \in \mathbb{Z}$ we can find integers $p_{0, n}, p_{1, n}, \ldots, p_{m, n}$ $(n \in \mathbb{N})$ such that

1. $\sum_{k=0}^{m} a_{k} p_{k, n} \neq 0$ for all $n \in \mathbb{N}$, 
2. $\lim _{n \rightarrow \infty}\left(p_{0, n} x^{k}-p_{k, n}\right)=0$ for $k=1,2, \ldots, m$.

Then $x$ is transcendent.

Proof. Suppose that $x$ is algebraic, then there exists a positive integer $m$ and integers $a_{0}, a_{1}, \ldots, a_{m}$ such that $\sum_{k=0}^{m} a_{k} x^{k}=0$. By adding and subtracting we find

$$
\sum_{k=0}^{m} a_{k}\left(p_{0, n} x^{k}-p_{k, n}\right)+\sum_{k=0}^{m} a_{k} p_{k, n}=0 .
$$

We know by assumption (1) that the integer $\sum_{k=0}^{m} a_{k} p_{k, n}$ is different from zero, hence in absolute value it is at least 1 . As a consequence

$$
\left|\sum_{k=0}^{m} a_{k}\left(p_{0, n} x^{k}-p_{k, n}\right)\right| \geq 1 \text {. }
$$

Taking $n \rightarrow \infty$, then assumption (2) gives a contradiction. Hence $x$ has to be transcendental.

Proving transcendence can therefore be done constructively by producing good simultaneous approximants to consecutive powers of $x$. Type II Hermite-Padé approximants can then be used in case $x^{j}=f_{j}(z)$ for a complete system of Markov functions $\left(f_{1}, f_{2}, \ldots, f_{m}\right)$. Hermite first showed that $\left(e^{c_{1} z}, e^{c_{2} z}, \ldots, e^{c_{r} z}\right)$ is a complete system for Hermite-Padé approximation near zero (rather than near $\infty$, which we described in this paper) whenever all $c_{i}$ are distinct complex numbers. He then used the type II Hermite-Padé approximants for the system $\left(e^{z}, e^{2 z}, \ldots, e^{m z}\right)$ to produce simultaneous rational approximants satisfying the conditions of Lemma 6.1. See [NS, pp. 130-131] or [VA2] for details of this proof.

\section{References}

[An] M. A. Angelesco, Sur deux extensions des fractions continues algébriques, C.R. Acad. Sci. Paris 18 (1919), 262-263.

[Ap] R. Apéry, Irrationalité de $\zeta(2)$ et $\zeta(3)$, Astérisque 61 (1979), 11-13.

[A1] A. I. Aptekarev, Asymptotics of simultaneously orthogonal polynomials in the Angelesco case, Mat. Sb. 136 (1988), 56-84 (in Russian); Math. USSR Sb. 64 (1989), 57-84.

[A2] A. I. Aptekarev, Multiple orthogonal polynomials, J. Comput. Appl. Math. 99 (1998), 423-447.

[A3] A. I. Aptekarev, Strong asymptotics of multiple orthogonal polynomials for Nikishin systems, Mat. Sb. (to appear).

[AMR] A. I. Aptekarev, F. Marcellán, I. A. Rocha, Semiclassical multiple orthogonal polynomials and the properties of Jacobi-Bessel polynomials, J. Approx. Theory 90 (1997), 117-146.

[AS] A. I. Aptekarev, H. Stahl, Asymptotics of Hermite-Padé polynomials, in 'Progress in Approximation Theory' (A. A. Gonchar, E.B. Saff, eds.), Springer-Verlag, Berlin, 1992, pp. $127-167$.

[B1] F. Beukers, A note on the irrationality of $\zeta(2)$ and $\zeta(3)$, Bull. London Math. Soc. 11 (1979), 268-272.

[B2] F. Beukers, Padé approximantions in number theory, in 'Padé Approximation and its Applications', Lecture Notes in Mathematics 888, Springer-Verlag, Berlin, 1981, pp. 9099.

[BE] P. Borwein, T. Erdélyi, Polynomials and Polynomial Inequalities, Springer-Verlag, Berlin, 1995.

[dB] M. G. de Bruin, Simultaneous Padé approximants and orthogonality, in 'Polynômes Orthogonaux et Applications' (C. Brezinski et al., eds.), Lecture Notes in Mathematics 1171, Springer-Verlag, 1985, pp. 74-83. 
[BL] J. Bustamante, G. López, Hermite-Padé approximation for Nikishin systems of analytic functions, Mat.Sb. 183 (1992), 117-138 (in Russian); Math. USSR Sb. 77 (1994), 367-384.

[DS1] K. Driver, H. Stahl, Normality in Nikishin systems, Indag. Math. (New Series) 5 (2) (1994), 161-187.

[DS2] K. Driver, H. Stahl, Simultaneous rational approximants to Nikishin systems, I, II, Acta Sci. Math. (Szeged) 60 (1995), 245-263; 61 (1995), 261-284.

[GR] A. A. Gonchar, E. A. Rakhmanov, On the convergence of simultaneous Padé approximants for systems of Markov type functions, Trudy Mat. Inst. Steklov 157 (1981), 31-48 (in Russian); Proc. Steklov Math. Inst. 3 (1983), 31-50.

[GRS] A. A. Gonchar, E. A. Rakhmanov, V. N. Sorokin, Hermite-Padé approximants for systems of Markov-type functions, Mat. Sb. 188 (1997), 33-58 (in Russian); Russian Acad. Sci. Sb. Math. 188 (1997), 671-696.

[K1] V. A. Kalyagin, On a class of polynomials defined by two orthogonality relations, Mat. Sb. 110 (1979), 609-627 (in Russian); Math. USSR Sb. 38 (1981), 563-580.

[K2] V. A. Kalyagin, Hermite-Padé approximants and spectral analysis of nonsymmetric operators, Mat. Sb. 185 (1994), 79-100 (in Russian); Math. USSR Sb. 82 (1995), 199-216.

[KR] V. A. Kaliaguine, A. Ronveaux, On a system of classical polynomials of simultaneous orthogonality, J. Comput. Appl. Math. 67 (1996), 207-217.

[KS] R. Koekoek, R.F.Swarttouw, The Askey-scheme of hypergeometric orthogonal polynomials and its q-analogue, Delft University of Technology, Report 98-17, 1998. Available on-line at http://ar.tri.tudelft.nl/ koekoek/research.html

[N1] E. M. Nikishin, A system of Markov functions, Vestnik Mosk. Univ., Ser. I (1979), no. 4, 60-63 (in Russian); Moscow Univ. Math. Bull. 34 (1979), 63-66.

[N2] E. M. Nikishin, On simultaneous Padé approximants, Math. Sb. 113 (1980), $499-519$ (in Russian); Math. USSR Sb. 41 (1982), 409-425.

[NS] E. M. Nikishin, V. N. Sorokin, Rational Approximations and Orthogonality, Translations of Mathematical Monographs 92, Amer. Math. Soc., Providence, RI, 1991.

[P] L. R. Pineiro, On simultaneous approximations for a collection of Markov functions, Vestnik Mosk. Univ., Ser. I (1987), no. 2, 67-70 (in Russian); Moscow Univ. Math. Bull. 42 (2) (1987), 52-55.

[S1] V. N. Sorokin, Simultaneous Padé approximants for finite and infinite intervals, Izv. Vyssh. Uchebn. Zaved., Mat. (1984) no. 8 (267), 45-52 (in Russian); J. Soviet Math. 28 (1984) no 8, 56-64.

[S2] V. N. Sorokin, A generalization of classical orthogonal polynomials and the convergence of simultaneous Padé approximants, Trudy Sem. Im. I. G. Petrovskogo 11 (1986), 125-165 (in Russian); Soviet Math. 45 (1989), 1461-1499.

[S3] V. N. Sorokin, A generalization of Laguerre polynomials and convergence of simultaneous Padé approximants, Uspekhi Mat. Nauk 41 (1986), 207-208 (in Russian); Russian Math. Surveys 41 (1986), 245-246.

[S4] V. N. Sorokin, Convergence of simultaneous Padé approximations to functions of Stieltjes type; Izv. Vyssh. Uchebn. Zaved., Mat. (1987), No.7 (302), 48-56 (in Russian); Soviet Math. 31 (1987) no. 7, 63-73.

[S5] V. N. Sorokin, Convergence of simultaneous Padé approximants for a class of functions, Mat. Sb. 132 (1987), 391-400 (in Russian); Math. USSR Sb. 60 (1988), 385-394.

[S6] V. N. Sorokin, Simultaneous Padé approximation for functions of Stieltjes type, Sib. Mat. Zh. 31, no. 5 (1990), 128-137 (in Russian); Sib. Math. J. 31, no. 5 (1990), 809-817.

[Sz] G. Szegö, Orthogonal Polynomials, Amer. Math. Soc. Colloq. Publ. 23, fourth edition 1975 .

[VA1] W. Van Assche, Orthogonal polynomials in the complex plane and on the real line, Fields Institute Comm. 14 (1997), 211-245.

[VA2] W. Van Assche, Approximation theory and analytic number theory, in 'Special Functions and Differential Equations' (K. Srinivasa Rao et al., eds.), Allied Publishers, New Delhi, 1998, pp. 336-355.

[VAY] W. Van Assche, S. B. Yakubovich, Multiple orthogonal polynomials associated with Macdonald functions, (manuscript). 
Department of Mathematics, Katholieke Universiteit Leuven, Celestijnenlaan 200 Building B, B-3001 Heverlee, Belgium

E-mail address: ralter@нis.kuleuven.ac.be 